\title{
Prognostic Indicators and Treatment Outcome in 94 Cases of Fibrolamellar Hepatocellular Carcinoma
}

\author{
Ahmed O. Kaseb ${ }^{a}$ Mohamed Shama ${ }^{a}$ Ibrahim Halil Sahin ${ }^{a}$ Ajay Nooka ${ }^{\mathrm{e}}$ \\ Hesham M. Hassabo ${ }^{a}$ Jean-Nicolas Vauthey ${ }^{b}$ Thomas Aloia $^{b}$ \\ James L. Abbruzzese ${ }^{a}$ Ishwaria M. Subbiah ${ }^{c}$ Filip Janku $^{d}$ Steven Curley ${ }^{b}$ \\ Manal M. Hassan ${ }^{a}$ \\ Departments of a Gastrointestinal Medical Oncology and ${ }^{\mathrm{b}}$ Surgical Oncology, ${ }^{\mathrm{c}}$ Division of Cancer Medicine and \\ ${ }^{\mathrm{d}}$ Department of Investigational Cancer Therapeutics (Phase I Program), The University of Texas MD Anderson Cancer \\ Center, Houston, Tex., and 'Department of Medical Oncology, Emory University School of Medicine, Atlanta, Ga., USA
}

\section{Key Words}

Fibrolamellar carcinoma $\cdot$ Hepatocellular carcinoma .

Recurrence-free survival · Survival

\begin{abstract}
Objective: Fibrolamellar hepatocellular carcinoma (FLHCC) is a rare variant of HCC. We report an analysis of the clinicopathologic features, treatment outcomes, and prognostic indicators of 94 cases. Methods: We retrospectively collected clinicopathologic and treatment outcome data from 94 FLHCC patients ( 48 males and 46 females). Median overall survival (OS) and recurrence-free survival (RFS) were calculated using Kaplan-Meier curves, and survival rates were compared by the log-rank test. The Cox proportional hazard model was used for univariate and multivariate estimation of hazard risk ratios and $95 \%$ confidence intervals $(\mathrm{Cl})$ for factors that correlated with survival and disease recurrence after resection. Results: Median age was 23 years (14-75); median OS was 57.2 months $(95 \% \mathrm{Cl}, 36.4-77.9)$, and median RFS was 13.9 months $(95 \% \mathrm{Cl}, 8.8-18.9)$. White race, female gender, early tumor stage, and tumor resection including metastasectomy were positively associated with longer OS,
\end{abstract}

while female gender was the only significant positive predictor of longer RFS. Finally, the 5-fluorouracil-interferon combination was the most frequently used systemic therapy. Conclusions: Our analyses indicate that surgical approaches including metastasectomy as the first-line treatment in FLHCC correlated with better outcome. Multimodality approaches, including neoadjuvant and adjuvant therapies, prolonged patient survival. Copyright $\odot 2013 \mathrm{~S}$. Karger AG, Basel

\section{Introduction}

The first description of fibrolamellar hepatocellular carcinoma (FLHCC) was in 1956 as a rare and distinct form of HCC [1]. However, the first clinical reports on this clinicopathologic entity and its favorable prognosis were published in the early 1980s [2, 3]. Unlike HCC, FLHCC has no sexual predominance, affects primarily younger patients, and typically is not associated with viral hepatitis or cirrhosis [4]. Notably, patients with FLHCC tend to have a better outcome than those with HCC, most likely because of their better liver condition and greater

\section{KARGER}

E-Mail karger@karger.com

www.karger.com/ocl
(C) 2013 S. Karger AG, Basel

0030-2414/13/0854-0197\$38.00/0
Ahmed O. Kaseb, MD

Department of Gastrointestinal Medical Oncology, Unit 426 The University of Texas MD Anderson Cancer Center 1515 Holcombe Boulevard, Unit 426, Houston, TX 77030 (USA)

E-Mail akaseb@mdanderson.org 
chances of successful surgical resection [3,5-9]. Several studies reported that prolonged survival associated with FLHCC was linked to greater feasibility of surgical resection, free surgical margins, and adequate lymph node dissection [10]; absence of vascular invasion or lymph node metastasis, and normal liver functions $[9,11]$. On the other hand, male sex, larger tumor size, and poor tumor differentiation have been found to correlate with poor prognosis in FLHCC [11] and are associated with a recurrence rate after resection as high as $50 \%[12,13]$. Because of the small number of cases reported, however, strong conclusions remain elusive, and, therefore, a lack of consensus on several aspects of FLHCC management remains a major challenge. For instance, while several reports have described the indolent nature of FLHCC and the superiority of surgical management in prolonging survival, few studies addressed the effect of neoadjuvant and adjuvant therapies on improving outcome. This is, in part, due to the conflicting data from studies using different regimens of chemotherapy in small patient cohorts.

We conducted a retrospective analysis of 94 consecutive patients with FLHCC treated at our institution. We examined their clinicopathologic features, treatment received, and predictors of clinical outcome. Our study comprises the largest single-institution series on FLHCC ever reported.

\section{Patients and Methods}

\section{Study Design and Study Population}

By searching the Tumor Registry Data of the University of Texas MD Anderson Cancer Center, we obtained a list of all patients with FLHCC who presented at our institution between 1992 and 2008. The protocol was approved by the Institutional Review Board of the MD Anderson Cancer Center. The diagnosis of FLHCC was confirmed for all patients by our pathologists. Structured data collection sheets were used and included demographic information, such as smoking, alcohol use, family history of cancer, medical history, and intake of hormones. A manual retrospective review of FLHCC patient records yielded clinical information such as disease stage, treatment approaches, chemotherapy regimens, and response to treatment.

The objective of this study was to collect all available clinical information regarding FLHCC patients, and analyze prognostic indicators and treatment outcomes. Each patient's disease stage was determined according to the American Joint Committee on Cancer (AJCC) TNM staging system of primary liver cancer [12].

Statistical Methods

We used the Statistical Package for Social Sciences (SPSS, Chicago, Ill., USA) program for data management and statistical analysis. Survival curves were generated by the Kaplan-Meier method, and the statistical significance of the differences was determined according to Gehan's modification of the Wilcoxon signed-rank test. The Cox proportional hazard model and multivariate analyses were used to test prognostic factors associated with overall (OS) and recurrence-free survival (RFS) in all patients. Variables tested included age, sex, race, stage of the disease, vascular invasion, lymph node involvement, presence of extrahepatic metastases, treatment modalities (surgery vs. chemotherapy vs. local therapies), and sequence of therapy. Assumptions of the Cox regression analyses were verified and all reported $\mathrm{p}$ values were two sided.

\section{Results}

\section{Patient Characteristics}

The total number of HCC cases that presented to our center between the years 1992 and 2012 was 1,882. Of these, 1,786 patients had HCC and 96 patients had FLHCC (5.1\%); 94 patients with pure FLHCC met the inclusion criteria, and 2 cases of FLHCC mixed with cholangiocarcinoma were excluded. The median age (range) of FLHCC patients was 23 years (range, 14-75), median OS was 57.2 months (95\% confidence interval, CI, 36.4-77.9 months); the Kaplan-Meier survival curve is shown in figure 1a. Forty-six of the 94 patients were females, and 7 were pregnant at the time of diagnosis. Most of the FLHCC cases were at an advanced stage at the time of initial diagnosis: AJCC stage III in 35 patients (38.2\%) and AJCC stage IV in 39 patients (42.3\%). Only 6 of 94 cases had evidence of cirrhosis (6.4\%). Patients and disease characteristics are summarized in table 1.

\section{Factors Predictive of OS}

We applied the univariate Cox regression analysis to our patient population to study the correlations between clinicopathologic factors and OS (table 2). Non-White race, multinodularity, and carcinomatosis were the most significant predictors of shorter survival in our patients. Other factors that tended to have a positive effect on survival, but did not have significant $\mathrm{p}$ values, included female sex $(p=0.1)$, absence of cirrhosis $(p=0.1)$, absence of lymph node metastasis $(\mathrm{p}=0.07)$, and absence of vascular invasion $(\mathrm{p}=0.4)$. Furthermore, exposure to treatment ever was the most significant predictor of longer survival in these patients. Cox regression multivariate analysis showed a significant association between carcinomatosis and poor survival: hazard ratio $(\mathrm{HR})=3.5$ (95\% CI: 1.2-10.1, p = 0.01). Furthermore, White patients tended to have better survival compared with non-White patients: $\mathrm{HR}=2.9$ (95\% CI: 0.9-9.7, $\mathrm{p}=0.08$ ). However, other factors included in table 2 showed no significant 


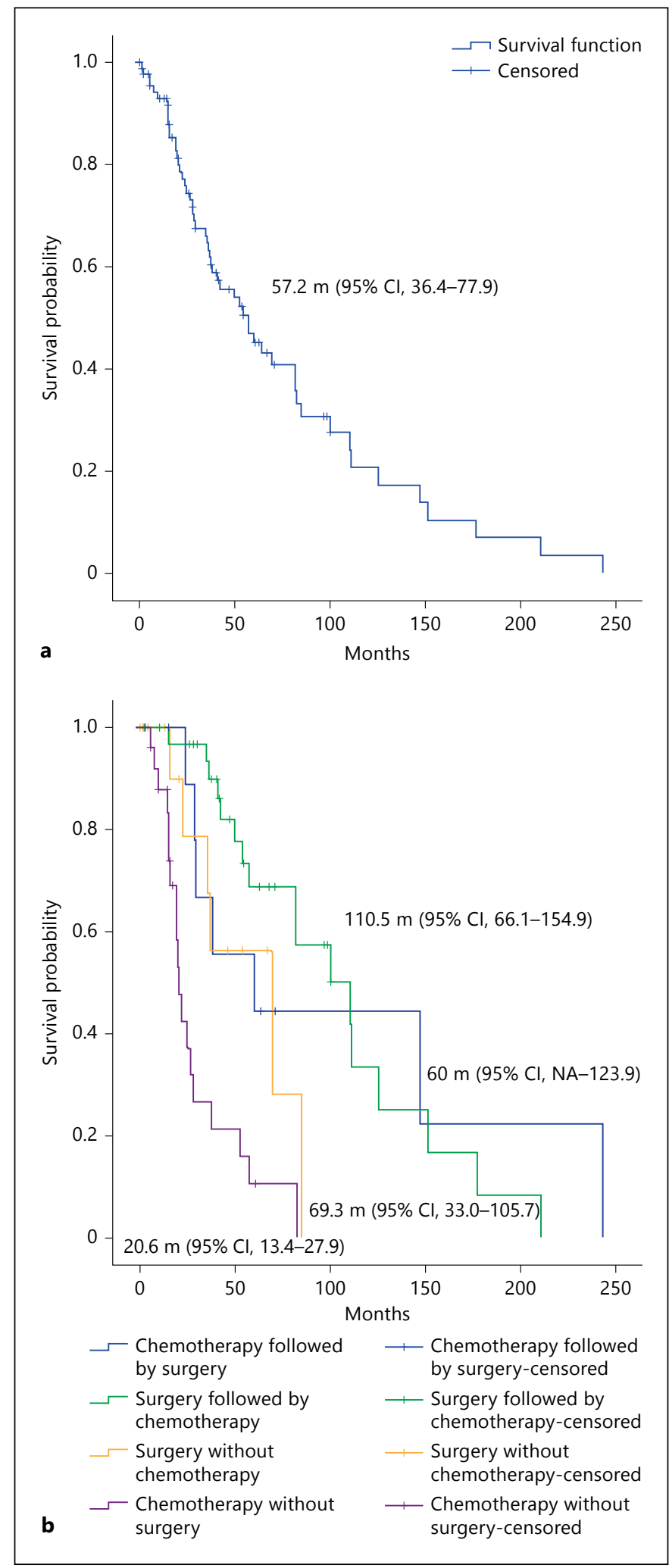

Fig. 1. a Kaplan-Meier OS estimate curve for all patients $(n=94)$. b Kaplan-Meier OS estimate curve for patients based on the treatment modality received. $\mathrm{m}=$ Months.

Outcome in FLHCC
Table 1. Demographic, epidemiological and clinical characteristics of the study population $(n=94)$

\begin{tabular}{|c|c|c|}
\hline Characteristics & $\mathrm{n}$ & $\%$ \\
\hline \multicolumn{3}{|l|}{ Sex } \\
\hline Male & 48 & 51.1 \\
\hline Female & 46 & 48.9 \\
\hline \multicolumn{3}{|l|}{ Race } \\
\hline White & 79 & 84.0 \\
\hline Hispanic & 8 & 8.5 \\
\hline African American & 6 & 6.4 \\
\hline Asian & 1 & 1.1 \\
\hline \multicolumn{3}{|c|}{ Hepatitis $\mathrm{C}$ virus infection } \\
\hline Reactive & 3 & 3.2 \\
\hline Nonreactive & 91 & 96.8 \\
\hline
\end{tabular}

Hepatitis B virus infection

Reactive

Nonreactive

Alcohol consumption

Yes

No

Cigarette smoking

Yes

$$
\text { No }
$$

History of diabetes mellitus

$$
\text { Yes }
$$

First degree history of cancer

$\begin{array}{lll}\text { Yes } & 28 & 29.8 \\ \text { No } & 66 & 70.2\end{array}$

Use of oral contraceptives

$$
\text { Yes }
$$

No

Pregnancy

$$
\text { Yes }
$$

No

AJCC staging

$$
\text { I }
$$

II

IIIA

IIIB

IIIC

IV

Vascular invasion

$$
\text { Yes }
$$

No

Lymph node metastasis

$$
\text { Yes }
$$

No

Carcinomatosis

Yes 13

No

Cirrhosis

$$
\text { Yes }
$$

3 
Table 2. Estimation of survival predictors: univariate analysis

\begin{tabular}{lcll}
\hline Variables & $\begin{array}{l}\text { 5-year } \\
\text { survival, \% }\end{array}$ & $\begin{array}{l}\text { Median } \\
\text { survival, months }\end{array}$ & $\begin{array}{l}\mathrm{p} \\
\text { value }\end{array}$ \\
\hline Male & $32(17-47)$ & $42.0(26.2-57.8)$ & 0.101 \\
Female & $60(41-75)$ & $84.9(62.9-06.9)$ & \\
White race & $48(34-59)$ & $57.2(44.6-69.7)$ & 0.017 \\
Non-White race & $21(4-48)$ & $20.6(5.9-35.4)$ & \\
No cirrhosis & $46(33-58)$ & $57.2(41.8-72.5)$ & 0.149 \\
Cirrhosis & $31(1-73)$ & $22.3(2.7-41.9)$ & \\
Single nodularity & $51(34-65)$ & $69.3(39.8-98.8)$ & 0.041 \\
Multinodularity & $30(12-50)$ & $26.4(9.9-42.9)$ & \\
Lymph node metastasis & & & \\
$\quad$ & $59(37-75)$ & $84.9(46.4-23.4)$ & 0.072 \\
$\quad$ Yes & $34(19-49)$ & $38.1(12.3-63.9)$ & \\
No vascular invasion & $61(30-82)$ & $69.3(34.7-03.9)$ & 0.072 \\
Vascular invasion & $42(27-57)$ & $54.2(28.4-80.7)$ & 0.416 \\
No carcinomatosis & $38(20-55)$ & $42.0(23.5-60.5)$ & 0.416 \\
Carcinomatosis & - & $24.9(1.3-48.6)$ & 0.019 \\
AJCC stage I-II & $76(32-89)$ & $84.9(33.5-36.3)$ & \\
AJCC stage III & $43(24-61)$ & $54.2(31.7-76.7)$ & 0.097 \\
AJCC stage IV & $36(20-53)$ & $28.9(15.3-2.59)$ & \\
\hline
\end{tabular}

95\% CI are shown in parentheses.

Table 3. Systemic chemotherapy regimens for FLHCC patients

\begin{tabular}{lcllllll}
\hline \multirow{2}{*}{ Regimen } & \multirow{2}{*}{ Total } & Adjuvant & \multicolumn{6}{l}{ Best response } \\
\cline { 3 - 8 } & & & CR & PR & MR & SD & PD \\
\hline 5-FU + IFN & 27 & 2 & 2 & 6 & 2 & 10 & 5 \\
PIAF & 10 & 2 & 0 & 0 & 1 & 6 & 1 \\
Xeloda + IFN & 10 & 1 & 0 & 0 & 3 & 3 & 3 \\
Other regimens & 6 & & & & & & \\
\hline
\end{tabular}

Numbers of patients are shown. The group of patients reported ( $\mathrm{n}=53$ ) includes patients who had neoadjuvant, adjuvant, or palliative therapy. Other regimens, 1 patient each: 5 -FU + IFN + cisplatin; 5 -FU + IFN + carboplatin; 5-FU + cisplatin + doxorubicin; 5-FU + bevacizumab; capecitabine; capecitabine + bevacizumab. PIAF = Cisplatin, IFN, doxorubicin, and 5-FU; CR = complete response; $\mathrm{PR}=$ partial response; $\mathrm{MR}=$ mixed response; $\mathrm{SD}=$ stable disease; $\mathrm{PD}=$ progressive disease.

impact on survival prediction. Table 3 includes chemotherapy regimens our patients received (53/94 patients), either in the neoadjuvant, adjuvant, or metastatic setting. Other systemic agents included sorafenib in 3 patients, and phase I trial agents in the form of proteasome inhibitor $(\mathrm{n}=1)$, liposomal doxorubicin + hepatic arterial infusion of cisplatin $(\mathrm{n}=1)$, pazopanib + trastuzumab $(\mathrm{n}=$
1), pazopanib + vorinostat $(\mathrm{n}=1)$, sunitinib + valproic acid $(\mathrm{n}=3)$, bevacizumab + sorafenib $(\mathrm{n}=1)$, and sirolimus + vorinostat $(\mathrm{n}=2)$.

Notably, interferon (IFN) regimens were the most commonly used (47/53 patients), with best tumor response occurring with a regimen of 5-fluorouracil (5-FU) combined with IFN. Furthermore, a serum $\alpha$-fetoprotein level above normal, although observed in only a few of the patients $(n=13)$, was a significant predictor of poor OS $(\mathrm{p}=0.04)$.

We also studied the treatment modalities and their sequencing for patients for whom such records were available. Metastasectomy was done in 18 cases and was found to be significantly associated with longer OS: the median OS was 145 months for those who underwent metastasectomy versus 35 months for those who did not $(p=0.0001)$. A total of 49 patients underwent surgical treatment with curative intent, and 34 of these had lymphadenectomy, which did not have a significant impact on survival. Interestingly, patients who had frontline surgery followed by adjuvant therapies $(\mathrm{n}=34)$ had the longest OS [110.5 months (95\% CI, 66.1-154.9)], and those who underwent neoadjuvant therapy followed by surgery $(n=10)$ had a median OS of 60 months (95\% CI, NA-123.9; p < 0.001; table 5; fig. 1b).

\section{Factors Predictive of RFS}

Of the 49 patients who underwent curative surgery, including 2 liver transplant cases, 5 had residual disease, and 41 patients had recurrent disease, indicating a recurrence rate of $83.7 \%$. Median RFS was 13.9 months (95\% CI, 8.8-18.9). Results of the Cox regression analysis of factors associated with RFS are summarized in table 5. Notably, baseline staging had no significant impact on RFS, while female sex was the only significant positive predictor of longer RFS (HR $=3.1 ; 95 \% \mathrm{CI}: 1.09-8.71, \mathrm{p}=$ $0.3)$. Additionally, there was a trend toward longer RFS in patients who had single tumors and no vascular invasion.

\section{Discussion}

This study reports a single-institution experience with 94 FLHCC cases, which represented $5.1 \%$ of the total number of HCC cases referred to the institution during the period from 1992 to 2012 . The availability of detailed medical records for our patients enabled a thorough analysis of prognostic indicators that correlated with OS and RFS after curative treatment approaches. 
Table 4. Log-rank analysis of OS in relation to treatment $(n=94)$

\begin{tabular}{lcccc}
\hline Management & \multirow{2}{*}{ Patients, $n$} & \multicolumn{2}{l}{ Survival, months } & \\
\cline { 3 - 5 } & & median & $95 \%$ CI & p value \\
\hline Surgery followed by adjuvant therapy & 34 & 110.5 & $66.1-154.9$ & \\
Neoadjuvant therapy followed by surgery & 10 & 60 & NA-123.9 & \\
Surgery without chemotherapy & 16 & 69.3 & $33.0-105.7$ & $<0.001$ \\
Chemotherapy without surgery $^{2}$ & 26 & 20.6 & $13.4-27.9$ & - \\
Local therapy alone $^{1}$ & 2 & 5.6 & - & \\
No treatment $^{1}$ & 6 & 1.8 & - & \\
\hline
\end{tabular}

${ }^{1}$ Undetermined $95 \% \mathrm{CI}$ due to the small number of patients. NA $=$ Not applicable.

Table 5. Cox regression analysis of factors associated with recurrence

\begin{tabular}{|c|c|c|c|c|c|}
\hline \multirow[t]{2}{*}{ Cox regression variables } & \multicolumn{2}{|c|}{$\begin{array}{l}\text { Recurrence, } \\
n \text { of patients }\end{array}$} & \multirow[t]{2}{*}{ RFS, months } & \multirow[t]{2}{*}{ HR (95\% CI) } & \multirow[t]{2}{*}{$\mathrm{p}$ value } \\
\hline & no & yes & & & \\
\hline Total & 8 & 41 & & & \\
\hline Male vs. female & $2 / 6$ & $19 / 22$ & $\begin{array}{l}12.2(8.9-15.3) \\
21.3(0.0-51.1)\end{array}$ & $3.1(1.09-8.71)$ & 0.03 \\
\hline Multi- vs. single nodularity & $2 / 4$ & $9 / 31$ & $\begin{array}{l}20.6(0.0-53.5) \\
13.7(10.7-16.6)\end{array}$ & $1.65(0.4-6.4)$ & 0.46 \\
\hline Vascular invasion vs. none & $3 / 2$ & $20 / 7$ & $\begin{array}{l}13.9(5.8-21.9) \\
17.1(5.1-29.4)\end{array}$ & $0.48(0.13-1.9)$ & 0.29 \\
\hline Positive lymph nodes vs. none & $3 / 3$ & $17 / 23$ & $\begin{array}{l}16.5(11.0-16.3) \\
13.7(8.9-24.3)\end{array}$ & $0.6(0.2-1.9)$ & 0.39 \\
\hline AJCC stage I + II & 4 & 10 & $17(6.5-27.3)$ & Reference & - \\
\hline AJCC stage III & 2 & 19 & $12(9.7-14.7)$ & $1.2(0.34-4.5)$ & 0.75 \\
\hline AJCC stage IV & 1 & 11 & $23.8(0.0-49.8)$ & $0.5(0.09-3.1)$ & 0.48 \\
\hline
\end{tabular}

Our results are consistent with and complimentary to previously reported observations regarding the clinicopathologic features of this rare disease and the advantages of surgical treatment approaches, including metastasectomy. Notably, patients also benefited from neoadjuvant and adjuvant multimodality therapy approaches. This is most likely related to disease down-staging among patients who presented initially at an advanced disease stage and eventually underwent surgical resection in addition to adjuvant local and systemic therapies. A recent study evaluated 30 cases of pure FLHCC [13] and reported a high incidence of metastasis especially to lymph nodes at presentation and upon relapse, which is consistent with our results (table 1). The role of chemotherapy in the neoadjuvant, adjuvant, and advanced tumor setting in FLHCC has not been studied vigorously, and the few published reports show conflicting results for different combinations of systemic chemotherapy applied to small numbers of FLHCC patients [13-16]. A recent multi-institution retrospective study of 95 FLHCC cases from three US centers reported a median OS of 6.7 years and indicated poor survival in females (median OS of 63.1 vs. 98.8 months in males, $\mathrm{p}=0.03$ ), advanced stage, unresectable disease, lymph node metastases, and macrovascular invasion [16]. While our study indicated a trend towards better outcome in fe- 
males as compared to males, with a longer median OS of 84.9 months for females versus 42 months for males ( $p$ $=0.10$ ), and a significantly longer recurrence-free interval of 21.3 months in females (12.2 months in males; $p$ $=0.03$ ), and a 5 -year survival rate of $60 \%$ in females versus $32 \%$ in males (table 2 ). However, our results of the favorable survival in females is consistent with a recent study that reported a 5 -year survival rate of $62 \%$ in females versus $42 \%$ in males [13]. Another recent study based on data from the Surveillance, Epidemiology, and End Results Program reported a series of 191 FLHCC cases, the largest to date, with a 5-year OS rate of 34\%, but no report of gender-specific rates, and revealed an interesting finding of two age-specific peaks for FLHCC, the first one in patients between 10 and 30 years and the second one in patients between 60 and 69 years, as compared to conventional HCC [17]. However, comparing results between studies of rare cancers has been challenging given the retrospective nature of collecting clinicopathologic data, in addition to the lack of detailed treatment history especially if collected from different institutions. Therefore, collaborative prospective studies in FLHCC are warranted to confirm these observations.

Notably, our previous report on the results of a prospective phase II clinical trial of systemic continuous 5 -FU and subcutaneous recombinant IFN $a-2 b$ for the treatment of HCC revealed that this regimen had promising activity in FLHCC cases [18]. The study included only 9 patients with FLHCC from a total of 43 HCC patients, but the 8 evaluable FLHCC patients had a median OS of 23 months: 1 patient had a complete response and 4 patients had a partial response. Our current analysis of a series of 27 patients treated with the same combination, in addition to 17 patients who received similar regimens (table 3), suggests potential benefits from the 5-FU-IFN chemotherapy combination in the neoadjuvant, adjuvant, and metastatic setting. This observation is interesting given the that one of the hallmark histopathologic features of FLHCC is the presence of laminated fibrous lay- ers, interspersed between cancer cells, and the potential anti-fibrotic effects of IFN $a-2 b$. However, prospective evaluation of this regimen is warranted.

Our study has several strengths, including the details on patient demographics, the large number of patients, which is the largest ever reported from a single institution, and the detailed multidisciplinary treatment history and follow-up. Limitations include being a retrospective analysis and the need to validate some of the results, such as gender variations in survival and treatment outcome.

In conclusion, progress in FLHCC management has been hampered by the lack of dedicated clinical trials, particularly with regard to systemic and local therapy approaches, in the neoadjuvant, adjuvant, and metastatic setting. Systemic and local therapies are particularly feasible for this patient population, given their lack of associated underlying chronic liver diseases that complicate HCC management. Such trials would be of great value in adding to our understanding of the natural history of FLHCC and improving the outcome of patients with this disease. One constraint on clinical trials of FLHCC is the small number of patients, which limits the significance of the results. This challenge highlights the importance of multicenter studies and international collaboration in this rare disease.

\section{Acknowledgment}

The study was supported by National Institutes of Health RO3 grant ES11481 and CA106458-01 (to M.M.H.).

\section{Disclosure Statement}

We have no commercial associations (e.g., consultancies, stock ownership, equity interests, or patent-licensing arrangements) that might pose a conflict of interest in connection with the present paper.

\section{References}

1 Edmondson HA: Differential diagnosis of tumors and tumor-like lesions of liver in infancy and childhood. AMA J Dis Child 1956;91: 168-186.

2 Berman MM, Libbey NP, Foster JH: Hepatocellular carcinoma. Polygonal cell type with fibrous stroma - an atypical variant with a favorable prognosis. Cancer 1980;46:14481455.
-3 Craig JR, Peters RL, Edmondson HA, Omata ML: Fibrolamellar carcinoma of the liver: a tumor of adolescents and young adults with distinctive clinico-pathologic features. Cancer 1980;46:372-379.

4 El-Serag HB, Davila JA: Is fibrolamellar carcinoma different from hepatocellular carcinoma? A US population-based study. Hepatology 2004;39:798-803.
5 Curutchet HP, Terez JJ, Kay S, Lawrence W Jr: Primary liver cancer. Surgery 1971;70:467-479.

6 el-Domeiri AA, Huvos AG, Goldsmith HS, Foote FW Jr: Primary malignant tumors of the liver. Cancer 1971;27:7-11.

-7 Malt RA, Van Vroonhoven TY, Kakumoto Y: Manifestations and prognosis of carcinoma of the liver. Surg Gynecol Obstet 1972;135:361364 
8 Moreno-Luna LE, Arrieta O, García-Leiva J, Martínez B, Torre A, Uribe M, León-Rodríguez E: Clinical and pathologic factors associated with survival in young adult patients with fibrolamellar hepatocarcinoma. BMC Cancer 2005;5:142.

9 Torbenson M: Review of the clinicopathologic features of fibrolamellar carcinoma. Adv Anat Pathol 2007;14:217-223.

10 Liu S, Chan KW, Wang B, Qiao L: Fibrolamellar hepatocellular carcinoma. Am J Gastroenterol 2009; 104:2617-2624, quiz 2625.

-11 Kakar S, Burgart LJ, Batts KP, Garcia J, Jain D, Ferrell LD: Clinicopathologic features and survival in fibrolamellar carcinoma: comparison with conventional hepatocellular carcinoma with and without cirrhosis. Mod Pathol 2005;18:1417-1423.
12 Greene FL, Page DL, Fleming ID, et al: AJCC Cancer Staging Manual, ed 6. New York, Springer, 2002.

13 Malouf GG, Brugières L, Le Deley MC, et al: Pure and mixed fibrolamellar hepatocellular carcinomas differ in natural history and prognosis after complete surgical resection. Cancer 2012;118:4981-4990.

14 Katzenstein HM, Krailo MD, Malogolowkin $\mathrm{MH}$, et al: Fibrolamellar hepatocellular carcinoma in children and adolescents. Cancer 2003;97:2006-2012.
El-Gazzaz G, Wong W, El-Hadary MK, et al: Outcome of liver resection and transplantation for fibrolamellar hepatocellular carcinoma. Transpl Int 2000;13(suppl 1):S406-S409.

16 Ang CS, Kelley RK, Choti MA, et al: Clinicopathologic characteristics and survival outcomes of patients with fibrolamellar carcinoma: data from the fibrolamellar carcinoma consortium. Gastrointest Cancer Res 2013;6:3-9.

17 Eggert T, McGlynn KA, Duffy A, et al: Epidemiology of fibrolamellar hepatocellular carcinoma in the USA, 2000-10. Gut 2013, E-pub ahead of print.

18 Patt YZ, Hassan MM, Lozano RD, et al: Phase II trial of systemic continuous fluorouracil and subcutaneous recombinant interferon Alfa-2b for treatment of hepatocellular carcinoma. J Clin Oncol 2003;21:421-427. 